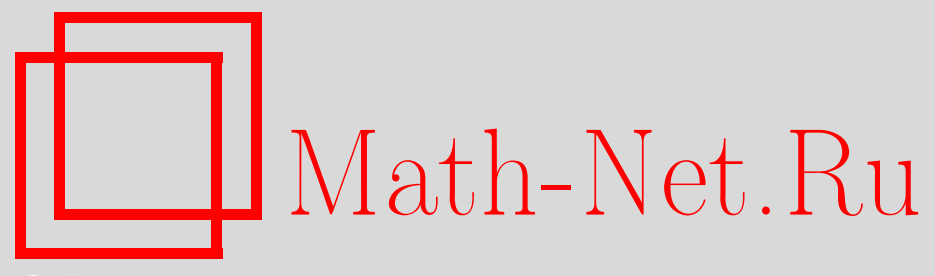

Б. Мукушев, Трансцендентные уравнения в задачах по физике, Квант, 2020, номер 8, 34-40

DOI: https://doi.org/10.4213/kvant20200803

Использование Общероссийского математического портала Math-Net.Ru подразумевает, что вы прочитали и согласны с пользовательским соглашением http://www . mathnet.ru/rus/agreement

Параметры загрузки:

IP: 54.92 .164 .108

26 апреля 2023 г., 13:58:36

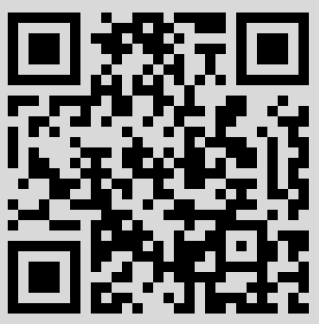




\section{рансцендентные уравнения в задачах по физике}

\section{Б.МУКУШЕВ}

РИ РАЗБОРЕ ЗАДАЧ ПО ФИЗИКЕ иногда приходится иметь дело с трансцендентными уравнениями, которые обычно не решаются аналитическим способом. Вот примеры трансцендентных уравнений:

$$
\begin{gathered}
2^{x}-10 x=0, \ln x-x^{2}=2 x, \\
0,7 \cos x-\sin 1,3 x=0 .
\end{gathered}
$$

Для решения таких видов уравнения используются методы приближенного вычисления: графический, численный, компьютерный.

Рассмотрим несколько задач из механики, при решении которых получаются трансцендентные уравнения и обсудим оптимальные методы решения подобных задач.

Задача 1. Под каким углом $\varphi$ к горизонту нужно бросить тело, чтобы за время его полета до возвращения на исходную высоту среднее значение модуля вектора тангении ального ускорения совпало с модулем среднего значения вектора нормального ускоре ния? Сопротивлением воздуха пренебречь.

Решение. Модуль вектора тангенциального ускорения $\vec{a}_{\tau}$ определяет изменение модуля скорости тела. Из соображений симметрии параболического движения тела рассмотрим только его подъем до наивысшей точки траектории. Для нахождения среднего значения модуля вектора тангенциального ускорения поделим общее изменение модуля скорости в процессе движения тела до максимальной высоты, равное $v-v_{0} \cos \varphi$, где $v_{0}$ - начальная скорость, на время подъема $T=\frac{v_{0} \sin \varphi}{g}$. Таким образом, среднее значение модуля вектора тангенциального ускорения равно

$$
\left\langle\left|\vec{a}_{\tau}\right|\right\rangle=\frac{v_{0}-v_{0} \cos \varphi}{\left(v_{0} \sin \varphi\right) / g}=g \frac{1-\cos \varphi}{\sin \varphi} .
$$

DOI: https://doi.org/10.4213/kvant20200803

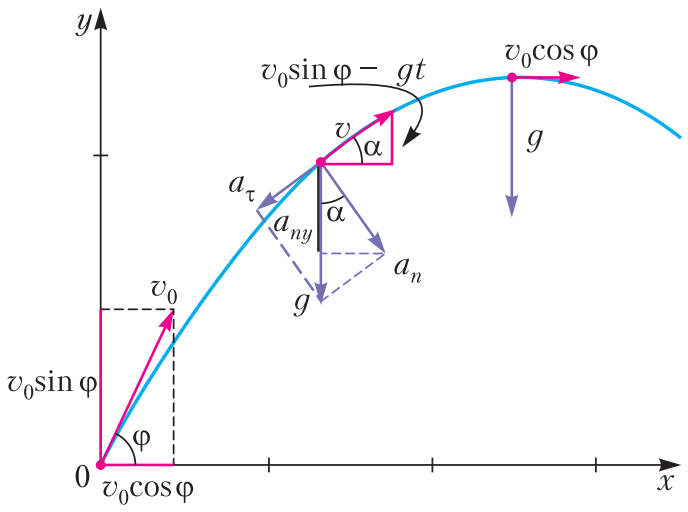

Pис. 1

Алгоритм расчета модуля среднего значения вектора нормального ускорения $\vec{a}_{n}$ выглядит по другому: сначала выполняется усреднение, а потом вычисляется модуль. В силу симметрии движения тела относительно вертикальной прямой, проходящей через наивысшую точку траектории, среднее значение вектора нормального ускорения будет направлено вертикально вниз. Поэтому можно усреднять его вертикальную проекцию $a_{n y}$ только за время подъема, так как за время спуска среднее значение проекции будет таким же:

$$
a_{n y}=a_{n} \cos \alpha=g \cos ^{2} \alpha,
$$

где $\alpha$ - угол наклона скорости к горизонту в момент времени $t$ от начала полета (рис.1). Итак, модуль среднего значения вектора нормального ускорения равен

$$
\left|\left\langle\vec{a}_{n}\right\rangle\right|=\left|\left\langle a_{n y}\right\rangle\right|=\frac{1}{T} \int_{0}^{T} g \cos ^{2} \alpha \cdot d t=\frac{1}{T} \int_{0}^{T} \frac{g d t}{\operatorname{tg}^{2} \alpha+1} .
$$

Поскольку $\operatorname{tg} \alpha=\frac{v_{0} \sin \varphi-g t}{v_{0} \cos \varphi}$, то

$$
\begin{aligned}
\left|\left\langle\vec{a}_{n}\right\rangle\right|= & \frac{1}{T} \int_{0}^{T} \frac{g d t}{\left(\frac{v_{0} \sin \varphi-g t}{v_{0} \cos \varphi}\right)^{2}+1}= \\
& =\left.\frac{v_{0} \cos \varphi}{T}\left(\frac{g t-v_{0} \sin \varphi}{v_{0} \cos \varphi}\right)\right|_{0} ^{T}=\frac{g \varphi \cos \varphi}{\sin \varphi} .
\end{aligned}
$$

По условию задачи $\left\langle\left|\vec{a}_{\tau}\right|\right\rangle=\left|\left\langle\vec{a}_{n}\right\rangle\right|$, или

$$
g \frac{1-\cos \varphi}{\sin \varphi}=\frac{g \varphi \cos \varphi}{\sin \varphi} \text {. }
$$




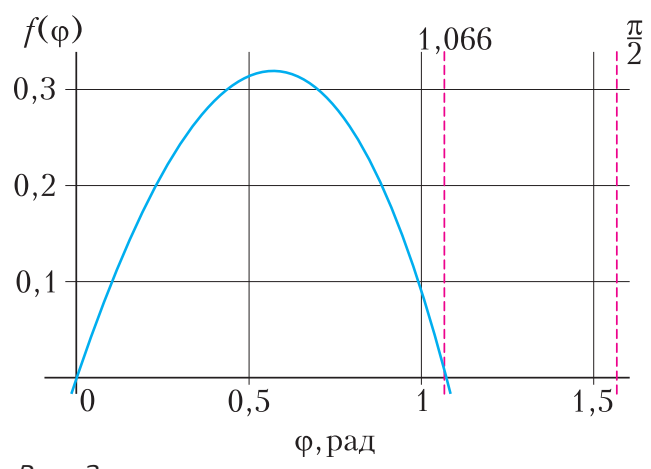

Pис. 2

Отсюда получаем трансцендентное уравнение

$$
\varphi \cos \varphi+\cos \varphi-1=0 .
$$

Одним из распространенных методов приближенного вычисления является графический метод, при его использовании получается оценочные результаты. Исследуем график функции

$$
f(\varphi)=\varphi \cos \varphi+\cos \varphi-1 .
$$

Если воспользуемся возможностями ППП MathCAD для построения графика этой функции, то можно улучшить точность результата решения трансцендентного уравнения. Из графика функции $f(\varphi)$ (рис.2), построенного посредством ППП MathCAD, можно видеть, что функция обратится в ноль приблизительно в точке

$$
\varphi \approx 1,066 \text { рад } \approx 61,12^{\circ} .
$$

Задача 2. Нить, обернутая вокруг неподвижного диска радиусом r, образует полуокружность (рис.3). Один конеи нити закреплен в точке $A$, ко второму конщу нити привязан грузик, который вначале удерживается в точке $B$ (точки $A$ и $B$ находятся на одной вертикали). В некоторый момент грузик отпускается. Какая часть нити
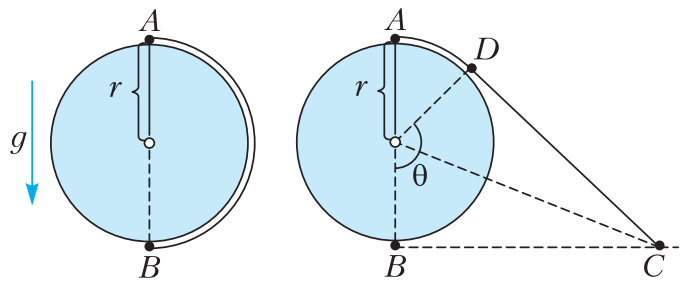

Pис. 3

Pис. 4 останется в соприкосновении с диском, когда грузик максимально удалится от начального положения? Трением пренебречь.

Решение. По закону сохранения энергии точка $B$ и точка максимального удаления $C$ находятся на одной горизонтали (рис.4). Из рисунка находим

$$
\operatorname{tg} \frac{\theta}{2}=\frac{C D}{r} .
$$

C другой стороны, длина дуги $B D$ равна длине отрезка $C D$ :

$$
\stackrel{\cup}{B D}=C D=r \theta \text {. }
$$

Итак,

$$
\operatorname{tg} \frac{\theta}{2}=\theta
$$

Это - трансцендентное уравнение, для его решения используем один из методов иисленного анализа. Чтобы определить число его решений на интересующем нас промежутке $0<\theta<\pi$, построим графики (рис.5)

$$
y=\theta \text { и } y=\operatorname{tg} \frac{\theta}{2} .
$$

Из графиков видно, что в интервале $0<\theta<\pi$ уравнение имеет единственный корень вблизи точки $\theta=2$.

Чтобы улучшить точность полученной оценки, воспользуемся методом последовательных приближений, который относится к численным методам. Для этого сначала запишем наше уравнение в виде функции

$$
\theta_{n}=f\left(\theta_{n-1}\right)=\operatorname{tg} \frac{\theta_{n-1}}{2} .
$$

Находим значения функции в нулевом приближении $\theta_{0}=2$ :

$$
\begin{aligned}
& \theta_{1}=f\left(\theta_{0}\right) \approx 1,557, \theta_{2}=f\left(\theta_{1}\right) \approx 0,986, \\
& \theta_{3}=f\left(\theta_{2}\right) \approx 0,537, \theta_{4}=f\left(\theta_{3}\right) \approx 0,275 .
\end{aligned}
$$

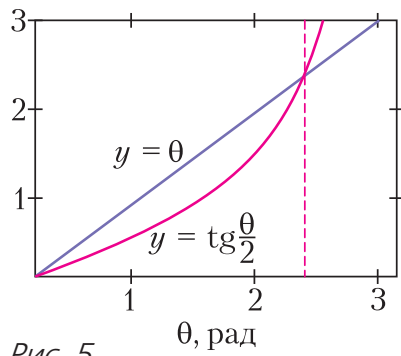

Pnc. 5 
Ясно, что последовательность все больше удаляется от предполагаемого значения корня. Значит, функция была выбрана неудачно, поэтому перепишем ее по другому:

$$
\theta_{n}=F\left(\theta_{n-1}\right)=2 \operatorname{arctg}\left(\theta_{n-1}\right) .
$$

Для значения аргумента $\theta_{0}=2$ найдем

$$
\begin{aligned}
& \theta_{1}=F\left(\theta_{0}\right) \approx 2,214, \theta_{2}=F\left(\theta_{1}\right) \approx 2,293, \\
& \theta_{3}=F\left(\theta_{2}\right) \approx 2,319, \theta_{4}=F\left(\theta_{3}\right) \approx 2,327, \\
& \theta_{5}=F\left(\theta_{4}\right) \approx 2,330, \theta_{6}=F\left(\theta_{5}\right) \approx 2,331, \\
& \theta_{7}=F\left(\theta_{6}\right) \approx 2,331 .
\end{aligned}
$$

Поскольку равенство $\theta_{6}=\theta_{7}$ выполнено с достаточной точностью, можно написать, что

$$
\theta \approx 2,331 \text { рад } \approx 133,6^{\circ} .
$$

Таким образом,

$$
\frac{\breve{A D}}{\cup}=\frac{\pi-\theta}{\pi}=0,258 .
$$

Задача 3. На закрепленный шар радиусом $R=20$ см сверху поставлена игрушка $\ll$ Ванька-встанька». Нижняя поверхность игруики - сферическая с иентром О и радиусом $r=5$ см, $C$ - центр тяжести игрушки, OC $=2$ см. До какого предельного угла $\beta_{\max }$ можно отклонить игрушку, чтобы она возвратилась в начальное положение?

Решение. При отклонении игрушки, например, вправо, центр тяжести смещается (рис.6). Если при этом он окажется левее вертикали $A B$, проходящей через точку опоры $A$, то момент силы тяжести будет возвра-

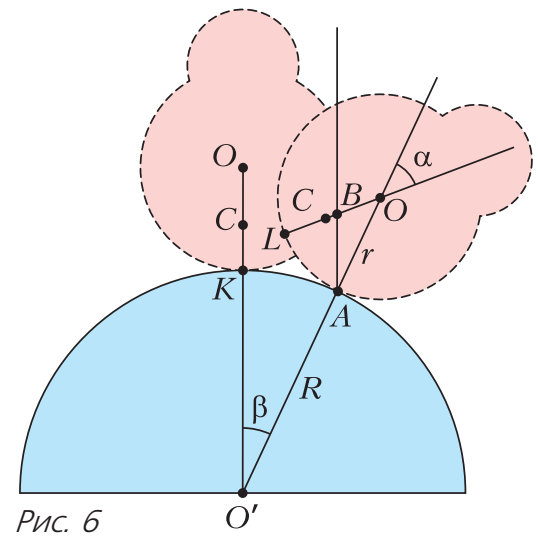

щать игрушку в положение равновесия. Если же центр тяжести окажется правее $A B$, игрушка упадет.

Обозначим $\alpha$ - угол поворота игрушки, $\beta$ угол поворота радиуса $O^{\prime} A$. Дуги $L A$ и $K A$ равны (нет проскальзывания), т.е. $\alpha r=\beta R$, откуда

$$
\alpha=\beta \frac{R}{r} .
$$

Из треугольника $O A B$ имеем

$$
\begin{aligned}
& \frac{O B}{\sin \beta}=\frac{A O}{\sin (\pi-\alpha-\beta)}=\frac{r}{\sin (\alpha+\beta)}= \\
&=\frac{r}{\sin (\beta(1+R / r))},
\end{aligned}
$$

или

$$
O B=\frac{r \sin \beta}{\sin (\beta(1+R / r))} .
$$

Игрушка возвратится в исходное положение, если $O C>O B$, т.е.

$$
O C>\frac{r \sin \beta}{\sin (\beta(1+R / r))} \text {. }
$$

С учетом соотношений $R / r=4$ и $\frac{r}{\mathrm{OC}}=\frac{5}{2}=2,5$ получим

$$
\sin 5 \beta>2,5 \sin \beta .
$$

Чтобы найти предельный угол $\beta_{\max }$, нам предстоит решить тригонометрическое уравнение

$$
\sin 5 \beta_{\max }=2,5 \sin \beta_{\max } .
$$

Это уравнение не имеет аналитического решения, т.е. его можно отнести к классу трансцендентных уравнений. Здесь мы воспользуемся компьютерным методом решения уравнения, в котором могут использоваться программы на языках Microsoft Excel, Matlab, Mathematica, Mathcad, Pascal, $\mathrm{C}^{++}$и др. Ниже предлагается решение уравнения $\sin 5 \beta-2,5 \sin \beta=0$ на языке программирования Pascal. При этом был использован один из методов численного анализа - метод Ньютона. Запишем программу: 


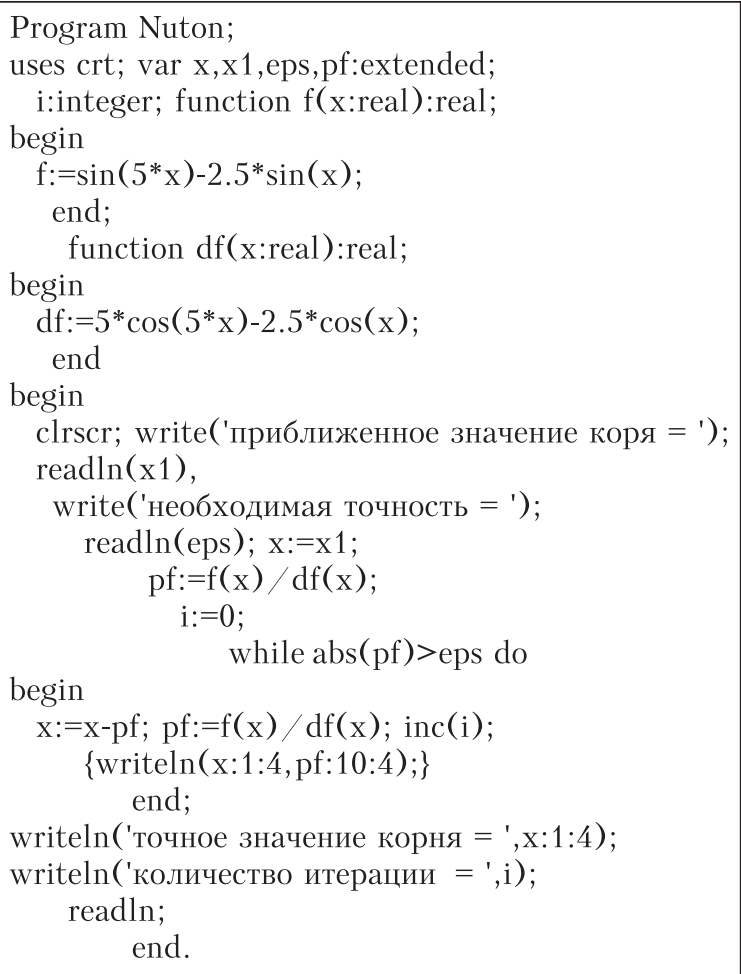

Результаты вычисления: приближенное значение корня - 1 , необходимая точность 0,0001 , значение корня с заданной точностью 0,3851 радиан, количество итераций -6 .

Итак,

$$
\beta_{\text {max }} \approx 0,3851 \text { рад } \approx 22,1^{\circ} .
$$

Значит, при $-22,1^{\circ}<\beta<22,1^{\circ}$ «Ванькавстанька» возвращается в начальное положение.

Задача 4. На неподвижном круглом полуцилиндре радиусом $R$ находится в равновесии однородная доска. Полуцилиндр расположен на горизонтальной плоскости (на столе). Толщина доски h, длина l, при этом $\frac{l}{h}=3, \frac{R}{h}=0,6$. Возвратится ли доска в начальное состояние при максимальном отклонении от горизонтального положения, когда доска коснется поверхности стола (рис.7)? Трение между иилиндром и доской велико.

Решение. Сначала нужно найти предельный угол отклонения доски $\varphi_{\text {пр }}$ от горизонтального положения, при меньших его зна-

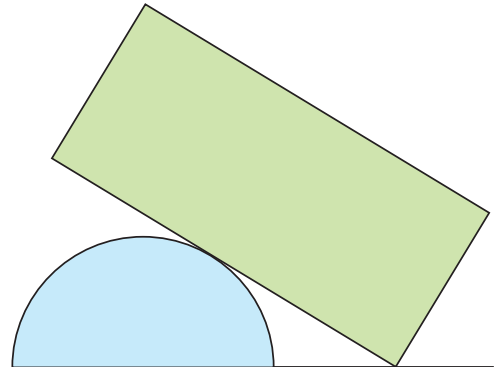

Рис. 7

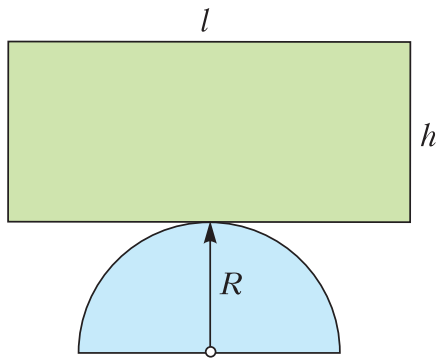

Pис. 8

чениях доска возвращается в начальное состояние. Для нахождения $\varphi_{\text {пр }}$ рассмотрим новую механическую систему, состоящую только из цилиндра и доски, когда отсутствует горизонтальная плоскость (рис.8). На первый взгляд может показаться, что равновесие доски вообще неустойчиво, так как центр масс доски лежит выше оси, вокруг которой она может поварачиваться. Однако здесь положение оси вращения не остается неизменным, поэтому этот случай требует специального исследования.

Поскольку доска уравновешена в горизонтальном положении, центр полукруга $O_{1}$ и центр тяжести доски $\mathrm{O}_{2}$ лежат на одной вертикали. Отклоним доску на некоторый угол $\varphi$ от горизонтали (рис.9). Точка опоры

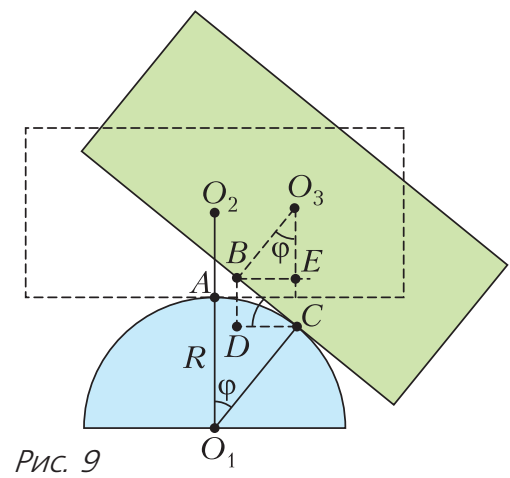


из положения $A$ перемещается в новое положение $C$, а та точка, которой доска до отклонения опиралась о полукруг, переходит в новое положение $B$. Поскольку проскальзывание отсутствует, длина дуги $A C$ равна длине отрезка $B C$ :

$$
\breve{A C}=B C=R \varphi .
$$

Центр тяжести доски $\mathrm{O}_{2}$ переходит в положение $\mathrm{O}_{3}$. Если вертикаль, проведенная через $\mathrm{O}_{3}$, проходит левее новой точки опоры $C$, то сила тяжести стремится вернуть доску в положение равновесия. Выразим это условие на языке математики. Проводим вертикаль через точку $B$ и видим, что должно быть выполнено условие $B E \leq D C$. Так как $B E=\frac{h}{2} \sin \varphi$, a $D C=B C \cos \varphi=R \varphi \cos \varphi$, получаем

$$
\frac{h}{2} \sin \varphi \leq R \varphi \cos \varphi, \text { или } \operatorname{tg} \varphi \leq \frac{2 R}{h} \varphi .
$$

Подставив $\frac{R}{h}=0,6$, получим $\operatorname{tg} \varphi \leq 1,2 \varphi$.

Итак, нам следует решать уравнение

$$
\operatorname{tg} \varphi-1,2 \varphi=0 .
$$

Данное уравнение трансцендентное. Посредством прикладной программы Mathcad построим график функции $y=\operatorname{tg} \varphi-1,2 \varphi$ на декартовых координатных осях (рис.10). Из графика видно, что корень, находящийся в интервале $0,5<\varphi<1$, имеет физический смысл. На рисунке 11 представлена программа MathCAD для реализации численного решения данного уравнения с начальным значением $\varphi=0,5$. У равнение решено посредством встроенной функции Find (най-

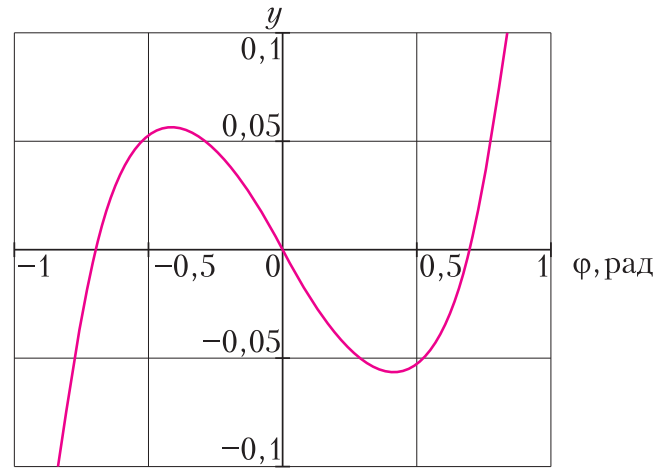

Рис. 10

\begin{tabular}{|cc|}
\hline$:=0.5$ "Начальное значение исходной \\
переменной \\
Given & "Ключевое слово \\
$\tan (\varphi)-1.2 \varphi=0$ & "Решаемое уравнение \\
Find $(\varphi)=0.695$ & "Решение уравнения \\
\hline
\end{tabular}

Рис. 11

ти): предельный угол отклонения доски равен

$$
\varphi_{\text {пр }} \approx 0,696 \text { рад } \approx 39,8^{\circ} .
$$

Переходим к условию нашей задачи. Из рисунка 12 находим

$$
\operatorname{tg} \varphi=\frac{O^{\prime} A}{A L}=\frac{O^{\prime} A}{B L-B A},
$$

где $B L=2,5 R, B A=K A=R \varphi$. Поэтому

$$
\begin{aligned}
\operatorname{tg} \varphi=\frac{R}{2,5 R-R \varphi}=\frac{1}{2,5-\varphi}, \text { или } \\
\qquad \operatorname{tg} \varphi-\frac{1}{2,5-\varphi}=0 .
\end{aligned}
$$

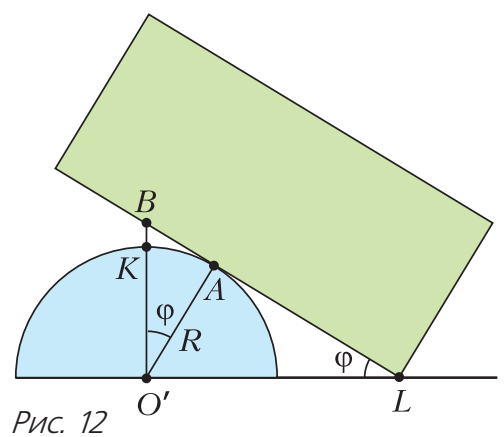

Опять получили трансцендентное уравнение. Для его решения, т.е. для оценки приближенного значения максимального угла отклонения доски, построим графики функций

$$
y=\operatorname{tg} \varphi, y=\frac{1}{2,5-\varphi} .
$$

Из графиков (рис.13) видно, что решение нашего уравнения лежит в интервале $\varphi \in(0,4 ; 0,6)$. Используем программу в среде Mathcad для реализации численного решения данного уравнения с начальным значением $\varphi=0,4$ (см. рис.11). Уравнение решено посредством встроенной функции Find (найти): максимальный угол отклонения 




РИс. 13

доски равен

$$
\varphi_{\text {max }} \approx 0,453 \text { рад } \approx 26^{\circ} .
$$

Значит,

$$
\varphi_{\max } \approx 26^{\circ}<\varphi_{\text {пр }} \approx 39,8^{\circ},
$$

поэтому доска возвратится в начальное положение.

Задача 5. На покоящийся, на гладкой поверхности кубик массой $m$, соединенный со стенкой пружиной, налетает справа второй кубик массой М (рис.14). Происходит абсолютно упругий удар, после чего первый кубик начинает колебаться. Какая часть периода колебаний пройдет до момента второго столкновения кубиков, если: а) $m / M=1 / 2$, б) $m / M \ll 1$ ? При каких значениях параметра $\gamma=m / M$ второе столкновение не произойдет вовсе?

Решение. Пусть $v_{0}-$ начальная скорость второго кубика, $v_{1}$ и $v_{2}-$ скорости кубиков после первого соударения, причем оба кубика двигаются влево. Из законов сохранения

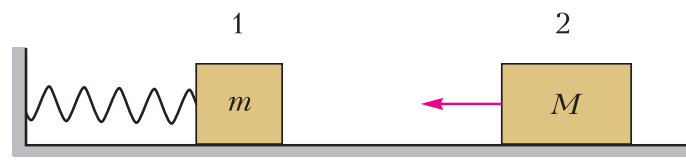

Pис. 14 импульса и энергии получаем

$$
M v_{0}=m v_{1}+M v_{2}, \frac{M v_{0}^{2}}{2}=\frac{m v_{1}^{2}}{2}+\frac{M v_{2}^{2}}{2},
$$

откуда

$$
v_{1}=v_{0} \frac{2}{1+\gamma}, v_{2}=v_{0} \frac{1-\gamma}{1+\gamma} .
$$

После соударения второй кубик будет двигаться равномерно, и его координата со временем будет изменяться по закону

$$
x_{2}=v_{2} t=v_{0} \frac{1-\gamma}{1+\gamma} t .
$$

Первый же кубик начнет колебаться по закону

$$
x_{1}=x_{m} \sin \omega t
$$

с частотой гармонических колебаний $\omega=\sqrt{\frac{k}{m}}$, где $k$ - жесткость пружины. Амплитуду колебаний $x_{m}$ найдем из закона сохранения энергии:

$$
\frac{m v_{1}^{2}}{2}=\frac{k x_{m}^{2}}{2} \text {, и } x_{m}=\frac{v_{1}}{\omega}=\frac{2 v_{0}}{(1+\gamma) \omega} .
$$

Таким образом,

$$
x_{1}=\frac{2 v_{0}}{(1+\gamma) \omega} \sin \omega t .
$$

Второе соударение кубиков произойдет при условии $x_{1}=x_{2}$, т.е.

$$
\frac{2 v_{0}}{(1+\gamma) \omega} \sin \omega t=v_{0} \frac{1-\gamma}{1+\gamma} t \text {. }
$$

Отсюда

$$
2 \sin \omega t=(1-\gamma) \omega t,
$$

или

$$
2 \sin \left(2 \pi \frac{t}{T}\right)=(1-\gamma) \cdot 2 \pi \frac{t}{T},
$$

где $-T$ период колебаний, $t / T-$ искомая часть периода.

Обозначим $\pi t / T=z$. Тогда предыдущее уравнение приобретет вид

$$
\sin 2 z=(1-\gamma) z .
$$

При $\gamma=1 / 2$ получаем

$$
\sin 2 z=\frac{z}{2} .
$$




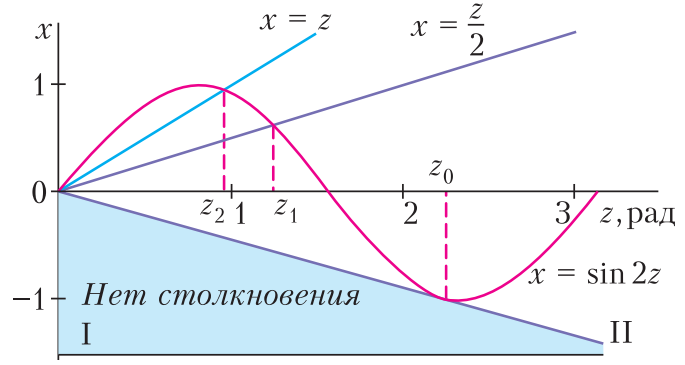

Pnc. 15

Начертим графики функций $x=\sin 2 z$ и $x=z / 2$ (рис.15). Из их пересечения видно, что результаты решения находятся в интервале $1<z<2$. Здесь используем один из методов приближенного вычисления - ме тод половинного деления. Составим программу в среде ППП MathCAD для рационального применения данного метода:
Второго соударения кубиков не произойдет, если их координаты больше не совпадут, т.е., говоря на языке графиков, если прямая, выражающая график зависимости координаты второго кубика от времени, не пересечет второй раз синусоиду. Этому соответствуют все прямые линии, выходящие из точки 0 и находящиеся в закрашенной области между линиями I и II (см. рис.15). Линия I соответствует случаю $m / M \rightarrow \infty$ (масса налетающего кубика очень мала). Осталось найти $\gamma$ для линии II - касательной к синусоиде.

Напишем общее уравнение касательной в виде

$$
f(z)=f^{\prime}\left(z_{0}\right)\left(z-z_{0}\right)+f\left(z_{0}\right)
$$

где $z_{0}$ - абсцисса точки касания этой прямой к синусоиде. Известно, что $f(z)=\sin 2 z$ и $f^{\prime}(z)=2 \cos 2 z$. Для точки 0 уравнение касательной записывается так:

\begin{tabular}{|c|c|}
\hline $\operatorname{bisec}(f, a, b, \varepsilon):=$ & $\begin{array}{l}f a \leftarrow f(a) \\
\text { while } b-a>\varepsilon \\
\mid \begin{array}{l}z \leftarrow \frac{a+b}{2} \\
f z \leftarrow f(z) \\
\text { (break }) \text { if } f z=0 \\
b \leftarrow z \text { if } f a \cdot f z<0 \\
a \leftarrow z \text { otherwise }\end{array} \\
z\end{array}$ \\
\hline $\begin{aligned} \mathrm{f}(\mathrm{z}) & :=\sin (2 \mathrm{z})-\frac{\mathrm{z}}{2} \\
\mathrm{a} & :=1 \\
\mathrm{~b} & :=1.5 \\
\varepsilon & :=0.001\end{aligned}$ & $\begin{array}{l}\text { ”Уравнение как функция пользователя } \\
\text { "Левая граница интервала } \\
\text { "Правая граница интервала } \\
\text { ”Погрешность уточнения корня }\end{array}$ \\
\hline $\operatorname{bisec}(f, a, b, \varepsilon)=1.237$ & "Уточненное значение корня уравнения \\
\hline
\end{tabular}

\section{Отсюда находим}

$$
\operatorname{tg} 2 z_{0}=2 z_{0} .
$$

Получено трансцендентное уравнение, его будем решать с помощью метода хорд, относящегося к методам приближенного вычисления. Программа, составленная в среде Паскаль или MathCAD, даст следующий результат: абсцисса точки касания касательной к
Таким образом,

$$
z_{1} \approx 1,237 \text { рад, } \pi \frac{t}{T} \approx 1,237, \frac{t}{T} \approx 0,39 .
$$

Если $m \ll M$, т.е. $\gamma \rightarrow 0$, то получаем уравнение

$$
\sin 2 z=z \text {. }
$$

ППП Mathcad даст такое решение:

$$
z_{2} \approx 0,948 \text { рад, или } \frac{t}{T} \approx 0,30 .
$$
синусоиде равна $z_{0} \approx 2,247$. Известно, что $\sin 2 z=(1-\gamma) z$, откуда

$$
\gamma=1-\frac{\sin 2 z_{0}}{z_{0}} \approx 1,434 \text {. }
$$

Итак, чтобы не произошло второго столкновения, должно выполняться условие

$$
1,434<\gamma<\infty \text {. }
$$

